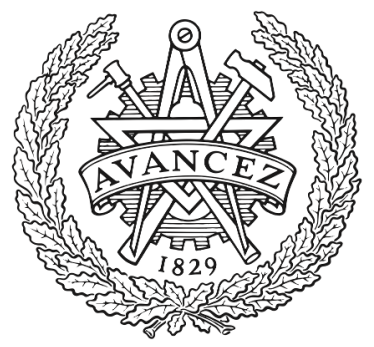

CHALMERS

UNIVERSITY OF TECHNOLOGY

\title{
Towards Adjoint-based Broadband Noise Minimization using Stochastic Noise Generation
}

Downloaded from: https://research.chalmers.se, 2023-04-26 06:12 UTC

Citation for the original published paper (version of record):

Zhou, B., Gauger, N., Yao, H. et al (2019). Towards Adjoint-based Broadband Noise Minimization using Stochastic Noise Generation. AIAA Scitech 2019 Forum.

http://dx.doi.org/10.2514/6.2019-0002

N.B. When citing this work, cite the original published paper. 


\title{
Towards Adjoint-based Broadband Noise Minimization using Stochastic Noise Generation
}

\author{
Beckett Y. Zhou* and Nicolas R. Gauger ${ }^{\dagger}$ \\ Chair for Scientific Computing, TU Kaiserslautern \\ Bldg 34, Paul-Ehrlich-Strasse, 67663 Kaiserslautern, Germany \\ Hua-Dong Yao; Shia-Hui Peng, ${ }^{\S}$ and Lars Davidson $₫$ \\ Department of Applied Mechanics, Chalmers University of Technology \\ SE-41296 Gothenburg, Sweden
}

\begin{abstract}
In this paper, we present an adjoint-based broadband noise minimization framework using stochastic noise generation (SNG). The SNG module is implemented in the open-source multi-physics solver suite SU2 and coupled with the existing Reynolds-averaged Navier-Stokes (RANS) to allow fast assessment of broadband noise sources. In addition, a discrete adjoint solver on the basis of algorithmic differentiation (AD) is developed for the coupled RANS-SNG system to enable efficient evaluation of broadband noise design sensitivities. The adjoint-based RANS-SNG framework developed in this work not only avoids the regularization problem that plagues the adjoint solutions for scale-resolving simulations, but also significantly lowers the computational cost and leads to a faster turn-around time for the initial design evaluation phase. Current results show that the RANS-SNG method can efficiently provide broadband noise level assessment for various configurations without resorting to computationally prohibitive scale-resolving simulations. Furthermore, current results also show that the AD-based coupled adjoint-RANS-SNG solver is highly accurate. Finally, shape optimizations performed on the basis of such coupled-sensitivity are shown to be effective in removing the broadband noise source in the trailing edge of a NACA0012 airfoil profile while maintaining aerodynamic performance imposed as an optimization constraint.
\end{abstract}

\section{Introduction}

Many aircraft noise sources exhibit broadband characteristics, which are typically generated by turbulent fluctuations in the flow and their interaction with the airframe. Some examples include: jet noise, trailing-edge noise where turbulent flow convects past a sharp trailing edge, and blade-wake interaction noise in rotor-craft applications where rotor/propeller blades are advanced into the turbulent wake generated by the preceding blades.

The most objectionable noise to the human ear occurs in the frequency range between 2 and $5 \mathrm{kHz}$. It is therefore essential to accurately characterize the broadband noise sources in order to facilitate noise-reducing designs that minimize the penalty of the high frequency weighting associated with perceived noise level. ${ }^{1}$ To that end, scale-resolving simulations such as direct numerical simulation (DNS), large-eddy simulation (LES) and variants of detached-eddy simulation (DES) have been shown to accurately capture noise sources containing a wide range of scales and can be used to study physical mechanisms behind new noise sources. ${ }^{2,3}$

From an optimal design perspective, the ability to simulate and predict the broadband noise is only half of the challenge - assessment from the computed aeroacoustic sources and far-field noise signature must be used to inform and iteratively update the design. That is, a sensitivity analysis of the noise metric with respect to the design variables must be performed. However, the exorbitant computational cost required by aforementioned scale-resolving simulations effectively precludes repeated evaluations necessary for finite-difference-type sensitivity analyses in each design cycle, especially for complex configurations with large numbers of design variables. To efficiently extract sensitivity

\footnotetext{
${ }^{*}$ Research Scientist, Member AIAA, yuxiang.zhou@scicomp.uni-kl.de

$\dagger$ Professor, Associate Fellow AIAA, nicolas.gauger@scicomp.uni-k1.de

${ }^{\ddagger}$ Research Scientist, Member AIAA, huadong.yao@chalmers.se

$\S$ Professor, Associate Fellow AIAA, peng@chalmers.se

ๆProfessor, lada@chalmers.se
} 
information to influence noise-reducing design, one turns to adjoint-based methods in which the computational cost of a sensitivity analysis approximately equals the cost of a single simulation and does not scale with the number of design parameters. ${ }^{4,5}$

However, it has been discovered in recent years that the chaotic turbulent contents in scale-resolving primal simulations lead to regularization issues ${ }^{6}$ in the resultant adjoint solution, causing it to gradually diverge. That is, even if adequate computing resources are available for the primal aeroacoustic simulations, which is questionable in itself for large complex configurations at high Reynolds numbers, the need for regularization presents a serious roadblock of a more fundamental nature for the adjoint problem.

While such regularization issues are not readily observed in simulations involving unsteady Reynolds-averaged Navier-Stokes equations (URANS), only the tonal noise component can be characterized by URANS, since RANSbased approaches do not aim to resolve transient turbulent structures (which contribute significantly to the broadband noise source), but model their effects based on the characteristics of the mean flow. As such, no time-dependent information on turbulent fluctuations responsible for broadband noise generation can be extracted. This has important ramifications on aeroacoustic optimizations. As shown in previous studies by Zhou et al. ${ }^{7}$ on interaction noise minimization of a rod-airfoil configuration, in which the noise source is computed by URANS, the optimizer is effective in removing the tonal noise component, leaving the broadband level largely unchanged.

The high computational cost of scale-resolving simulations, compounded by the regularization issues in solving the accompanying adjoint system, constitutes an urgent need for a more cost-effective alternative capable of providing broadband noise assessment within the timescales acceptable to the design cycles in the aircraft industry. Clearly, a middle-ground between the RANS-based approach and scale-resolving simulations needs to be sought.

To that end, the stochastic noise generation (SNG) method pioneered by Béchara et al. ${ }^{8}$ and Bailly et al. ${ }^{9}$ appears to be a suitable candidate. This method is based on the assumption that the unsteady turbulent velocity field can be constructed as a sum of plane waves whose wave numbers, intensities and phases are selected in such a way so as to match the mean statistical properties of the turbulent flow. As the first step, a steady RANS simulation is performed on the configuration to extract the local turbulence kinetic energy and other statistical quantities, which can be either the turbulence dissipation rate or the specific turbulence dissipation rate. Next, random Fourier modes are used to synthesize the unsteady turbulent velocity field with the same statistical characteristics of local turbulence kinetic energy, time scales and length scales as the preceding RANS solution. The turbulent field thus generated is then used for the evaluation of source terms of the linearized Euler equations (LEE) ${ }^{9}$ or acoustic perturbation equations (APE), ${ }^{10}$ which propagates sound from the source region to the far-field. Improved versions have been presented in the works of Billson et al., ${ }^{11}$ Casalino and Barbarino, ${ }^{12}$ and di Francescantonio et al. ${ }^{13}$ Industrially relevant applications to evaluate the flap side-edge noise and main landing gear noise have been demonstrated by Yao et al. ${ }^{14,15}$

It should be emphasized here that the RANS-SNG methodology developed in this work is not meant to predict the broadband noise to an absolute level but rather to provide the characterization or the trend of the broadband noise for efficient adjoint-based design optimization. Indeed, the adjoint-based RANS-SNG framework proposed here not only avoids the regularization problem that plagues the adjoint solutions for scale-resolving simulations, but more importantly it also significantly lowers the computation cost and leads to a faster turn-around time for the initial design evaluation phase.

In this work, we couple the RANS-SNG method described above with a discrete-adjoint solver developed on the basis of algorithmic differentiation (AD), replacing the laborious and error-prone hand-differentiation of the discretized governing equations. This however, is not the only advantage of AD-based adjoint. By construction, AD-based adjoints are accurate to machine precision as they do not incur any roundoff or truncation error. In addition, the frozen turbulence assumption typically used in many URANS-based adjoint formulations is eliminated, since the turbulence models while not analytically differentiable are still algorithmically differentiable. The application of AD also leads to robust and consistent adjoints in that the adjoint solver inherit the same convergence properties as the primal solver. Lastly, if the operator overloading $\mathrm{AD}$ method is used with expression template technique in $\mathrm{C}++,{ }^{16}$ the resultant discrete adjoint framework offers extra flexibility - the adjoint solver can be automatically updated with primal code modification and one can easily define any objective function from any state variable. This is an extremely attractive characteristic for unsteady optimization problems in the multidisciplinary setting using a suite of multi-physics solvers where the objective function may be different depending on the type of problems being addressed. AD-based discrete adjoint has been applied to steady aerodynamic shape design problems by Hovland et al. ${ }^{17}$ and Gauger et al. ${ }^{18}$ More recently, it was successfully applied by Nemeli et al. ${ }^{19}$ in the unsteady setting to the active flow control of an industryrelevant high-lift configuration HIREX from Airbus.

A natural question that arises is whether the optimizations performed based on the RANS-SNG framework can provide reliable design updates - is a noise-minimized design determined by a RANS-SNG-based optimization still 
considered optimal when analyzed with high-fidelity turbulence resolving simulations or even experimental measurements? In this work, to shed some light on this question, we perform adjoint-based noise optimization using the RANS-SNG framework and compare the baseline and optimized designs using scale-resolving simulations.

The remainder of this paper is organized as follows. In Section II, the RANS-SNG method as well as the optimization framework based on discrete adjoint and AD are presented. Section III presents numerical results while the conclusion and outlook for future work are discussed in Section IV.

\section{Unsteady Aerodynamic and Aeroacoustic Optimization Framework}

\section{II.A. Multi-Physics Solver SU2}

The Stanford University Unstructured (SU2) open source software suite was specifically developed for solving problems governed by partial differential equations (PDEs) and PDE-constraned optimization problems. It was developed with the aerodynamic shape optimization problems in mind. Therefore the suite is centered around a RANS solver capable of simulating compressible, turbulent flows commonly found in problems in aerospace engineering. The governing equations are spatially discretized using the finite volume method, on unstructured meshes. A number of convective fluxes discretization schemes have been implemented, such as the Jameson-Schimdt-Turkel (JST) scheme and the upwind Roe scheme. The turbulence can be either modeled by the Spalart-Allmaras(S-A) model or the Menter Shear Stress Transport (SST) Model.

For the sake of conciseness, details regarding the formulations and implementations of the SU2 solver suite will not be presented in this paper. Further details such as mesh deformation, dynamic mesh movement, multigrid implementations, validation and verification cases, as well as the continuous adjoint framework developed in tandem with SU2, the readers are referred to the published work by Palacios et al. ${ }^{20}$ of the SU2 team. In the next section we direct the attention of the readers to a new coupled RANS-SNG broadband noise assessment framework developed for the current work.

\section{II.B. RANS-SNG Broadband Noise Assessment Framework}

The method of stochastically reconstructing the noise-generating unsteady turbulent velocity field based on turbulence statistics computed in a preceding RANS solution was proposed by Kraichnan and further developed by Béchara et al. and Bailly and Juvé. In essence, a space-time turbulent velocity field can be expressed as a sum of random Fourier modes,

$$
\vec{u}(\vec{x}, t)=2 \sum_{n=1}^{N_{F}} \hat{u}_{n} \cos \left[\vec{k}_{n} \cdot(\vec{x}-\vec{U} t)+\psi_{n}\right] \vec{\sigma}_{n}
$$

where $\hat{\vec{u}}_{n}, \vec{k}_{n}, \psi_{n}$ and $\vec{\sigma}_{n}$ are statistical velocity magnitude, wave number vector, phase and direction associated with the $n^{\text {th }}$ Fourier mode, convecting in a mean velocity $\vec{U} \cdot{ }^{21,22}$

The vector $\vec{k}_{n}$ is generated randomly on a sphere with radius $k_{n}$, in order to ensure isotropy of the generated velocity field. The two polar angles $\varphi_{n}$ and $\theta_{n}$ that define the $n^{\text {th }}$ wave number vector $\vec{k}_{n}$ comply with the probability densities as follows:

$$
\begin{gathered}
P\left(\varphi_{n}\right)=(2 \pi)^{-1}, \quad-\pi / 2 \leq \varphi_{n} \leq \pi / 2 \\
P\left(\theta_{n}\right)=\cos \left(\theta_{n}\right) / 2, \quad-\pi / 2 \leq \theta_{n} \leq \pi / 2
\end{gathered}
$$

To keep divergence free, the velocity field satisfies the following condition:

$$
\vec{k}_{n} \cdot \vec{\sigma}_{n}=0
$$

This requires that the velocity vector $\vec{\sigma}_{n}$ lie in a plane orthogonal to $\vec{k}_{n}$. It is assumed that $\vec{\sigma}_{n}$ is uniformly distributed in the plane. The angle $\alpha_{n}$ between $\vec{\sigma}_{n}$ and $\vec{k}_{n}$ is with the uniform probability density as

$$
P\left(\alpha_{n}\right)=(2 \pi)^{-1}, \quad-\pi \leq \alpha_{n} \leq \pi
$$

The phase vector $\psi_{n}$ is uniformly distributed as well,

$$
P\left(\psi_{n}\right)=(2 \pi)^{-1}, \quad-\pi \leq \psi_{n} \leq \pi
$$


The wavenumbers are associated with the frequencies following the relationship:

$$
k=\frac{2 \pi f}{c_{0}}
$$

where $k$ is the modulus of $\vec{k}, f$ denotes the frequency, and $c_{0}$ is the speed of sound. In this work the frequency range of $20 \mathrm{~Hz} \leq f \leq 5000 \mathrm{~Hz}$ is studied. The speed of sound is set to $340 \mathrm{~m} / \mathrm{s}$.

The magnitude $\hat{\vec{u}}_{n}$ of each mode is computed so that the turbulence energy spectrum $E\left(k_{n}\right)$ correspond to the energy spectrum for isotropic turbulence, giving:

$$
\hat{u}_{n}=\sqrt{E\left(k_{n}\right) \Delta k_{n}}
$$

where $\Delta k_{n}$ is the band of the wave number $k_{n}$, and $E$ denotes the energy spectrum. This way the sum of the squares of $\hat{\vec{u}}_{n}$ over all $n$ is equal to the total turbulence kinetic energy

$$
K=\sum_{n=1}^{N_{F}} \hat{u}_{n}^{2}
$$

The energy spectrum is assumed in the form of Von Kármán-Pao isotropic turbulence spectrum as

$$
E(k)=\frac{2 A}{3} \frac{K}{k_{e}}\left(\frac{k}{k_{e}}\right)^{4} \exp \left[-2\left(\frac{k}{k_{\eta}}\right)^{2}\right]\left[1+\left(\frac{k}{k_{e}}\right)^{2}\right]^{(-17 / 6)}
$$

where $A$ is constant, $K$ is the turbulence kinetic energy, $k_{e}$ is the wavenumber of the maximum energy, and $k_{\eta}=$ $\varepsilon^{1 / 4} v^{-3 / 4}$ is the wavenumber of the Kolmogorov scale. The constants $A$ and $k_{e}$ are determined by the energy and length of the integral scale. The quantities are computed based on the RANS solutions of $K$ and $L_{T}=c_{1} u^{13} / \varepsilon$, where $u^{\prime}=\sqrt{2 K / 3}$. It gives $A \simeq 1.453$ and $k_{e}=0.747 / L_{T}$. The constant $c_{1}$ is a tuning parameter in this model. It adjusts the length scale $u^{\prime 3} / \varepsilon$ to the integral scale of the turbulence. The constant is close to one, but it should be optimized according to the practical flow conditions. In the present work, $c_{1}$ is set to one by default.

In the traditional application of such RANS-SNG method, at this point wave equations such as LEE or APE are typically solved using the mean flow field computed by RANS with source terms evaluated using the turbulent velocity field generated above. The solution of the wave equations thus gives the propagation of sound from the source region to the surrounding far-field.

In this work, as a first step, we elect to evaluate the Lighthill's stress tensor $T_{i j}$ from the turbulent field synthesized in the SNG step. The design objective for the adjoint solver is formulated in terms of $T_{i j}$ as a measure of the broadband noise source. In particular:

$$
\mathscr{J}^{B B N}=\left\|\frac{1}{V_{s}} \frac{1}{N_{t}} \sum_{m=1}^{N_{x}} \sum_{n=1}^{N_{t}} \mathbf{T}\left(\vec{x}_{m}, t_{n}\right) \Delta V_{m}\right\|^{F r o b}
$$

where $\mathbf{T}=T_{i j}=\rho u_{i} u_{j}$ is the first term of the Lighthill's stress tensor, $u_{i}$ is the turbulent velocity field synthesized in the SNG step, $V_{s}=\sum_{m=1}^{N_{x}} \Delta V_{m}$ is the total volume in the user-defined source region, $N_{x}$ is the number of volume elements in the source region, and $N_{t}$ is the total number of time steps of $\vec{u}$ constructed in the SNG step.

\section{II.C. AD-based Discrete Adjoint Framework}

The implementation of the discrete adjoint formulation in this work is eased by the use of automatic differentiation $(\mathrm{AD})^{\mathrm{a}}$, eliminating the error-prone hand-differentiation of the discretized equations. AD was developed based on the observation that any simulation code, regardless of its complexity is merely a sequence of elementary operations whose differentiation rules are well known. Therefore, by successive applications of the chain-rule through the computer program, it is possible to compute both the simulation output and its derivative with respect to prescribed design variables simultaneously. A remarkable feature of $\mathrm{AD}$, owing to its construction, is that it does not incur any truncation errors compared to the traditional finite difference method. In particular, the derivatives are accurate to machine accuracy. This is a very attractive characteristic of $\mathrm{AD}$, since accurate evaluation of the gradient requires exact differentiation of the fixed point iterator $G$ as evidenced by Equations 26 and 27 in the following discussion.

Next we present the AD-based discrete adjoint framework developed in the context of the SU2 solver described in Section II.A. The vector of design variables $\alpha$ defines the geometry of the design configuration and can be chosen

${ }^{a}$ performed using $\mathrm{AD}$ tool $\mathrm{CoDiPack}^{23}$ 
as the amplitudes of Hicks-Henne functions ${ }^{24}$ in $2 \mathrm{D}$ or as the control points of the Free-Form deformation method ${ }^{25}$ for 3D problems. According to a movement of the surface based on the current values of the design variables, a mesh deformation routine using the Linear Elasticity method creates a new mesh $X$. The solver then evaluates the state variable $U$ and the objective function $J$. Using this setting, the optimization problem incorporating a steady state constraint can be written as

$$
\begin{array}{cc}
\min _{\alpha} & J(U(\alpha), X(\alpha)) \\
\text { subject to } & R(U(\alpha), X(\alpha))=0
\end{array}
$$

where $R(U)$ is the spatially-discretized residual vector. Note that $R(U)$ might not only include the flow residual but also residuals of other coupled models. In case of the RANS equations plus a turbulence model we have

$$
U:=\left(\begin{array}{c}
U_{f} \\
U_{t}
\end{array}\right), \quad R(U)=R\left(U_{f}, U_{t}\right):=\left(\begin{array}{c}
R_{f}\left(U_{f}, U_{t}\right) \\
R_{t}\left(U_{f}, U_{t}\right)
\end{array}\right)
$$

The application of dual-time stepping method then solves the following problem through a fictitious time $\tau$ to converge to a steady state solution:

$$
\frac{d U}{d \tau}+R(U)=0
$$

Further assume the implicit Euler method is used to time march the above equation to steady state.

$$
U^{n+1}-U^{n}+\Delta \tau R\left(U^{n+1}\right)=0, \quad n=1, \ldots, N
$$

The resultant nonlinear system can be linearized around $U^{n}$ to solve for the state $U^{n+1}$

$$
U^{n+1}-U^{n}+\Delta \tau\left[R\left(U^{n}\right)+\left.\frac{\partial R}{\partial U}\right|^{n}\left(U^{n+1}-U^{n}\right)\right]=0, \quad n=1, \ldots, N
$$

This can be written in the form of a fixed-point iteration:

$$
U^{n+1}=G\left(U^{n}\right), \quad n=1, \ldots, N
$$

where $G$ represents an iteration of the pseudo time stepping. By the Banach fixed-point theorem, recurrence (18) converges a stationary point if $G$ is contractive, i.e. if $\left\|\frac{\partial G}{\partial U}\right\|<1$ in a suitable matrix norm. The fixed point iteration then converges to the numerical solution $U^{n}=U^{*}$ :

$$
R\left(U^{*}\right)=0 \Leftrightarrow U^{*}=G\left(U^{*}\right) .
$$

Then the optimization problem can be posed as:

$$
\begin{array}{cll}
\min _{\alpha} & & J(U(\alpha), X(\alpha)) \\
\text { subject to } & U(\alpha) & =G(U(\alpha), X(\alpha)) \\
& X(\alpha)=M(\alpha) .
\end{array}
$$

We can define the Lagrangian associated to this problem as

$$
\begin{aligned}
L(\alpha, U, X, \bar{U}, \bar{X}) & =J(U, X)+[G(U, X)-U]^{T} \bar{U}+[M(\alpha)-X]^{T} \bar{X} \\
& =N(U, \bar{U}, X)-U^{T} \bar{U}+[M(\alpha)-X]^{T} \bar{X}
\end{aligned}
$$

where $N$ is the shifted Lagrangian

$$
N(U, \bar{U}, X):=J(U, X)+G^{T}(U, X) \bar{U} .
$$

If we differentiate $L$ with respect to $\alpha$ using the chain rule, we can choose the adjoint variables $\bar{X}$ and $\bar{U}$ in such a way, that the terms $\frac{\partial U}{\partial \alpha}$ and $\frac{\partial X}{\partial \alpha}$ can be eliminated. This leads to the following equations for $\bar{U}$ and $\bar{X}$ :

$$
\begin{aligned}
& \bar{U}=\frac{\partial}{\partial U} N(U, \bar{U}, X)=\frac{\partial}{\partial U} J^{T}(U, X)+\frac{\partial}{\partial U} G^{T}(U, X) \bar{U} \\
& \bar{X}=\frac{\partial}{\partial X} N(U, \bar{U}, X)=\frac{\partial}{\partial X} J^{T}(U, X)+\frac{\partial}{\partial X} G^{T}(U, X) \bar{U}
\end{aligned}
$$


Finally, the derivative of the Lagrangian, that is, the total derivative of $J$, reduces to

$$
\frac{\mathrm{d} L^{T}}{\mathrm{~d} \alpha}=\frac{\mathrm{d} J^{T}}{\mathrm{~d} \alpha}=\frac{\mathrm{d}}{\mathrm{d} \alpha} M^{T}(\alpha) \bar{X} .
$$

Equation (26) is a fixed-point equation in $\bar{U}$ and can be solved in the style of the flow solver using the iteration

$$
\bar{U}^{n+1}=\frac{\partial}{\partial U} N\left(U^{*}, \bar{U}^{n}, X\right)
$$

once we have found a numerical solution $U=U^{*}$ of equation (18). Since $G$ is a contractive function if the flow solver has reached a certain level of convergence (i.e. $\left\|\frac{\partial G}{\partial U}\right\|<1$ in some suitable matrix norm), also $\frac{\partial N}{\partial U}$ will be contractive since

$$
\left\|\frac{\partial}{\partial \bar{U}}\left[\frac{\partial N}{\partial U}\right]^{T}\right\|=\left\|\left[\frac{\partial G}{\partial U}\right]^{T}\right\|=\left\|\frac{\partial G}{\partial U}\right\|<1 .
$$

Thus, it directly inherits the convergence properties of the flow solver. Note that in this work, the derivative terms on the right-hand side of flow adjoint (Eqn 26) and mesh adjoint (Eqn 27) equations are computed using AD. The effect of the state variables in the source region $V_{s}$ on the stochastically generated noise source and thus the broadband noise design objective $J$ is 'transmitted' through the term $\frac{\partial}{\partial U} J^{T}(U, X)$. That is, the sensitivity of the broadband noise source with respect to the mean flow field computed by RANS is expressed as $\frac{\partial}{\partial U} J^{T}(U, X)$, which is accumulated to the flow adjoint iterator at each sub-iteration in evaluating the coupled adjoint of RANS-SNG. The computational chain of the coupled RANS-SNG broadband noise assessment and noise-adjoint framework is outlined on Figure 1.

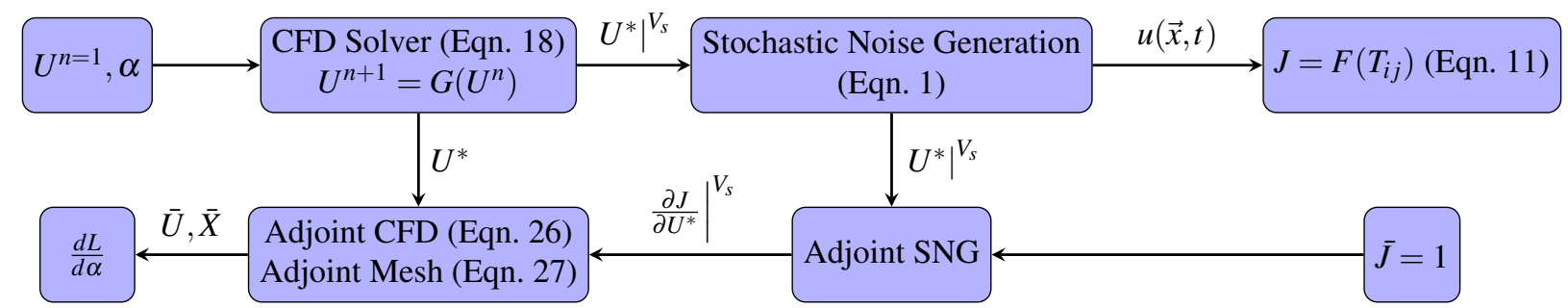

Figure 1: Computational chain of the coupled RANS-SNG broadband noise assessment and noise-adjoint framework

\section{Results}

\section{III.A. Broadband Noise Assessment with RANS-SNG Method}

The RANS-SNG method is applied to assess the broadband noise level of a 2-D NACA0012 airfoil at $M_{\infty}=0.2$, $R e_{c}=6.0 \times 10^{6}$ and $A o A=8^{\circ}$. The RANS solution for this test case, computed with SST $k-\omega$ turbulence model has been validated against experiment in the earlier work of Palacios et al. ${ }^{20}$ Turbulence kinetic energy (TKE) and specific dissipation rate $\omega$ are extracted from RANS solution in the focus region defined by: $x \in[0.8,1.5], y \in[-0.1,0.15]$, shown as the rectangular box on Figure 2. SNG and associated sensitivities are computed within the frequency range from 1 to $5 \mathrm{kHz}$. A total of $N_{t}=1000$ snapshots of the turbulent velocity field are stochastically generated and subsequently used to compute the first term of the Lighthill's stress tensor $\left(T_{i j}=\rho u_{i} u_{j}\right)$. Figure 3 shows the three components of the time-averaged Lighthill's stress tensor. The diagonal components clearly dominate in this case. Figure 4 shows the same three components at a lower angle of attack of 4 degrees which shows much weaker noise sources. This agrees with physical intuition - a larger angle of attack leads to a wider turbulent wake, which in turn gives rise to stronger quadrupole sources. 


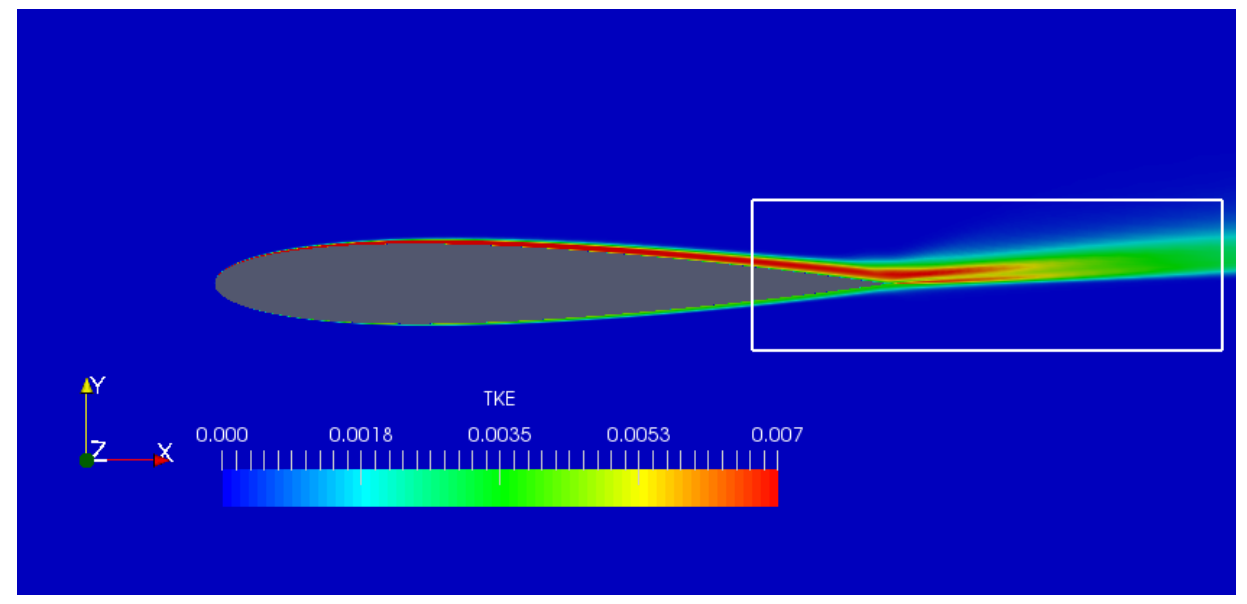

(a)

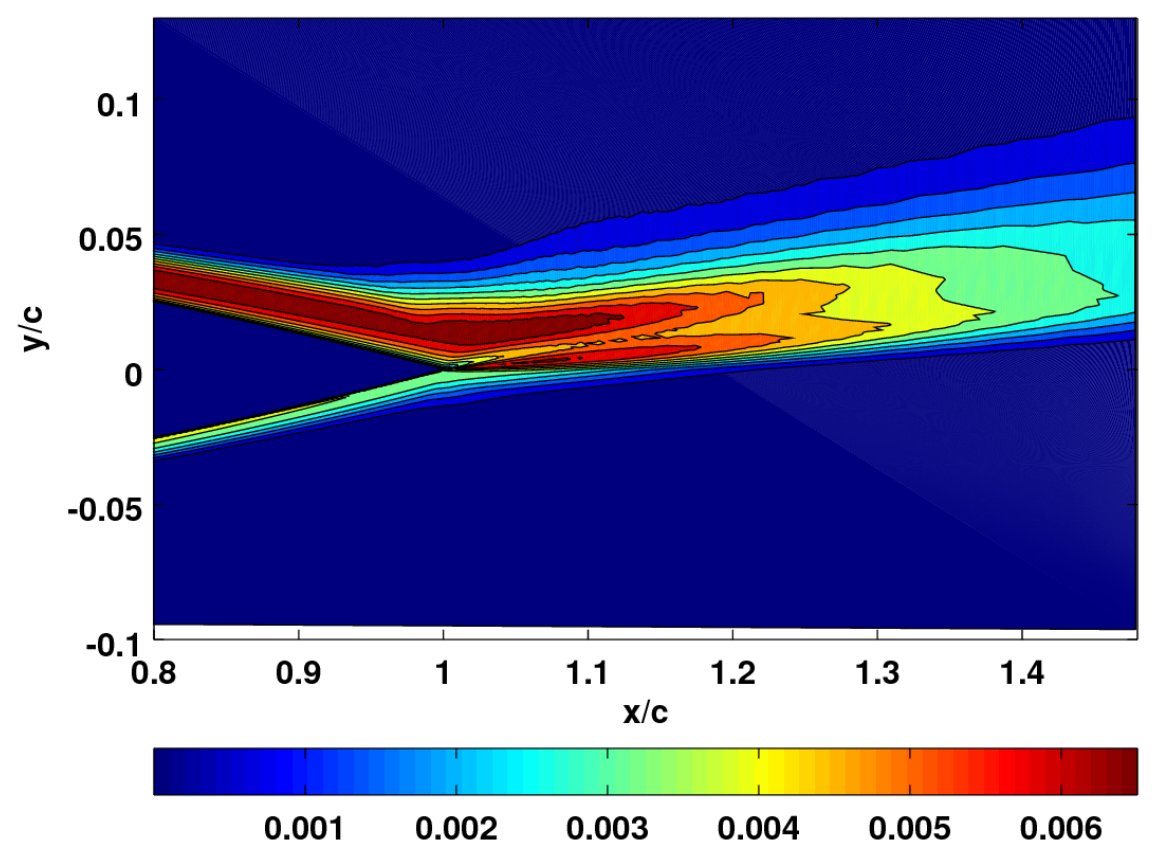

(b)

Figure 2: (a) Turbulence kinetic energy of a NACA0012 profile at $8^{\circ}$ angle of attack, $R e=1.0 \times 10^{6}$ and $M=0.15$, computed by a RANS calculation in SU2. The white lines enclose the focus region $\left(V_{s}\right)$ in which the SNG is applied to evaluate the broadband noise source. (b) Zoom-in view of the focus region in the wake. 


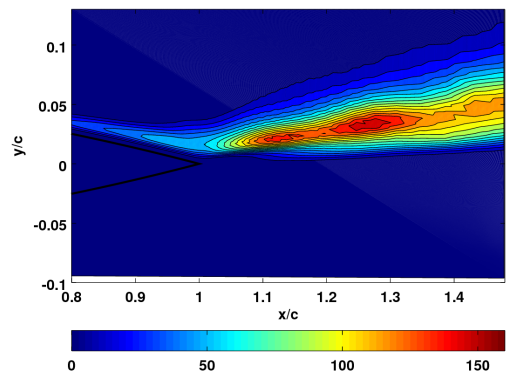

(a) $\bar{T}_{1,1}$

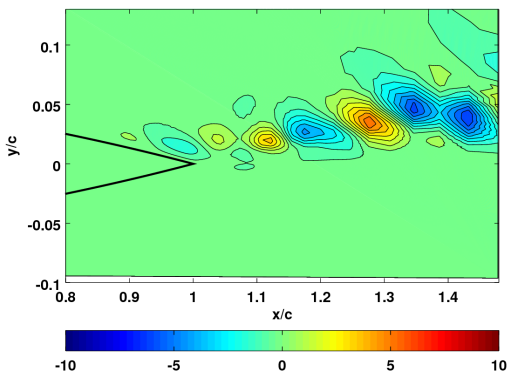

(b) $\bar{T}_{1,2}$

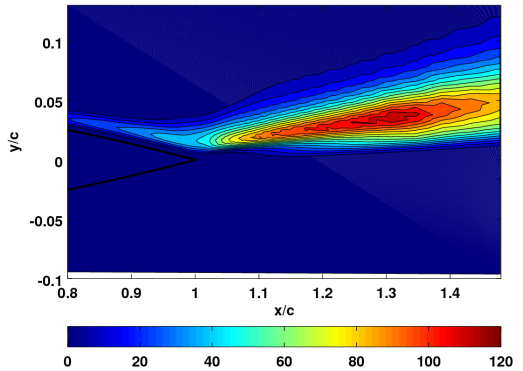

(c) $\bar{T}_{2,2}$

Figure 3: Three components of the time-averaged Lighthill's stress tensor at angle of attack of 8 degrees

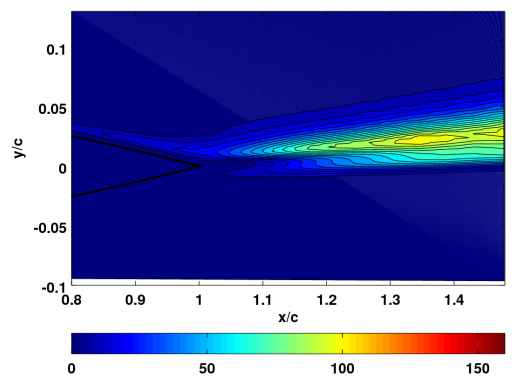

(a)

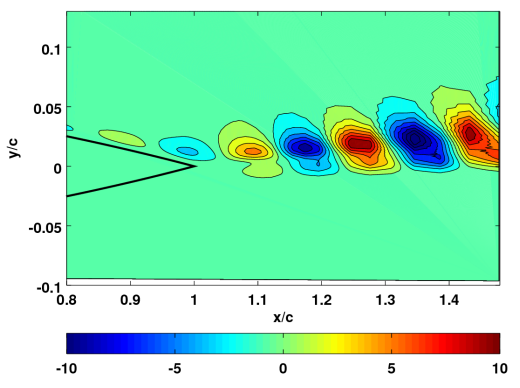

(b)

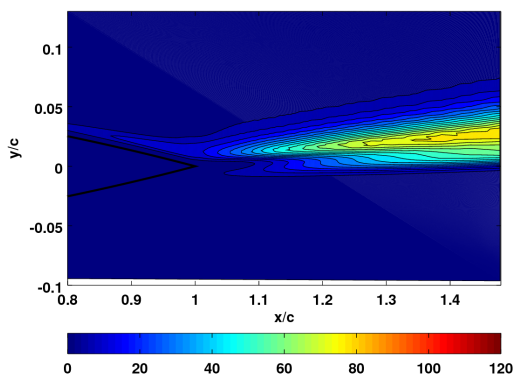

(c)

Figure 4: Three components of the time-averaged Lighthill's stress tensor at angle of attack of 4 degrees

\section{III.B. Validation of Noise Adjoint}

The SNG module, with TKE and $\omega$ as input and $\mathscr{J}^{B B N}$ (Eqn. 11) as output, is differentiated using AD. Figure 1 compares the the maximum sensitivities with respect to the two input variables within the focus region $\left(V_{s}\right)$, computed by AD and finite difference. The agreement is excellent.

It is interesting to examine the distributions of TKE, broadband noise design objective and its sensitivity with respect to TKE. As shown on Figure 11, while the peak TKE region, as expected resides in the turbulent boundary layer, the broadband noise source (the Frobenius-norm of the time-averaged Lighthill's stress tensor), as well as the peak sensitivity regions are all located further downstream in the wake region. It therefore would not be effective for an optimization framework to directly target the high-TKE regions within the boundary layer. Instead, a shape optimization should be conducted to morph the shape so as to reduce the TKE in the wake, where the strong quadrupole sources are. To that end, the effect of the turbulent variables on the noise source must be coupled with the shape design variables (which controls the distribution of the turbulent flow variables) through the flow adjoint, as outlined in Section II.C.

The coupled adjoint sensitivity is validated against finite difference gradient, as shown on Figure 6. In this case the design variables $x$ are the 18 Hicks-Henne bump functions ( 9 on each surface) used to parameterize the airfoil geometry. The agreement as shown on the figure, is excellent.

\begin{tabular}{|c|c|c|}
\hline & $\max \left(\frac{\partial \mathscr{I}^{B B N}}{\partial K}\right)$ & $\max \left(\frac{\partial \mathscr{I}^{B B N}}{\partial \omega}\right)$ \\
\hline \hline Adjoint-Mode AD & $4.785054967 \mathrm{E}+1$ & $1.426646088 \mathrm{E}-3$ \\
\hline Finite Difference & $4.785054950 \mathrm{E}+1$ & $1.426646095 \mathrm{E}-3$ \\
\hline
\end{tabular}

Table 1: Comparison of peak sensitivity values between adjoint-mode $\mathrm{AD}$ and finite difference $\left(\delta=10^{-6}\right)$ computations. 


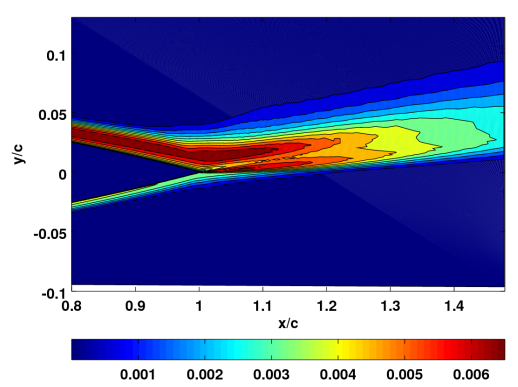

(a) $T K E$

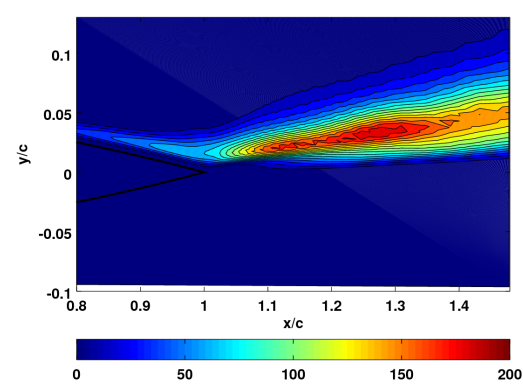

(b) || $\bar{T}||^{F r o b}$

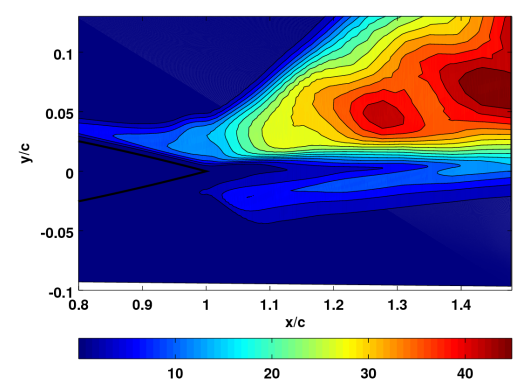

(c) $\frac{\partial \mathscr{J}^{B B N}}{\partial K}$

Figure 5: Distributions of TKE (a), broadband noise design objective (b), and its sensitivity with respect to TKE (c)

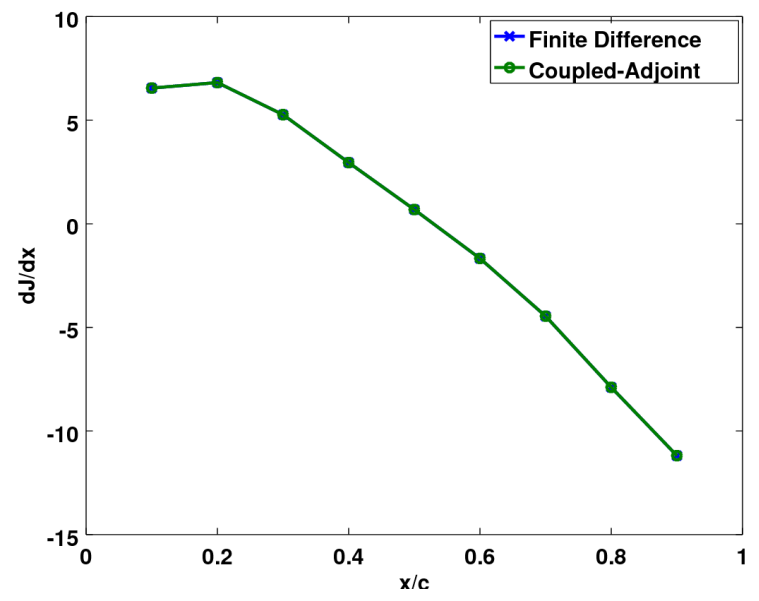

(a) Pressure Side

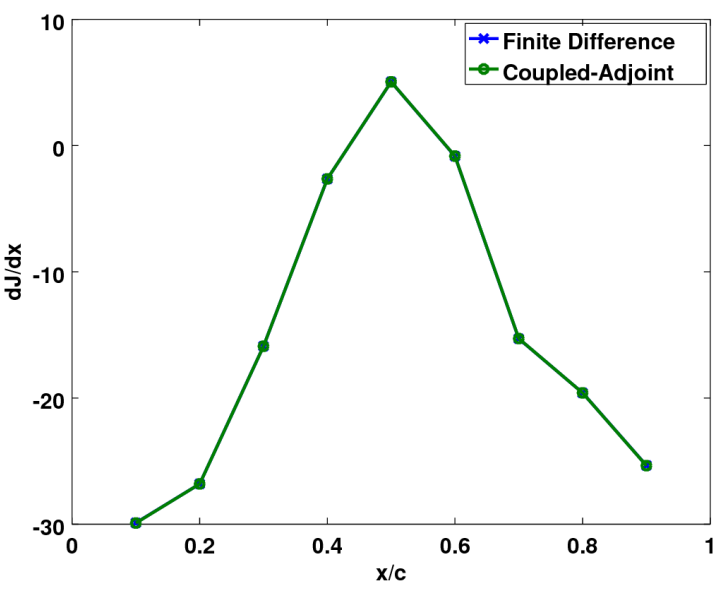

(b) Suction Side

Figure 6: Comparison between the gradients computed using finite difference (second order central difference with a step size of $10^{-6}$ ) and adjoint mode of $\mathrm{AD}$, at various chordwise locations on the airfoil surface 


\section{III.C. Broadband Noise Source Minimization, Without Aerodynamic Constraints}

Having validated the coupled RANS-SNG adjoint, a broadband noise source minimization is performed for 35 design iterations using SU2. The Reynolds number and angled of attack are reduced to $R e_{c}=1.0 \times 10^{5}$ and $A o A=5^{\circ}$ respectively. The former reduction is intended to make the aeroacoustic analyses of the baseline and optimized designs using scale-resolving simulations, such as large-eddy simulation more computationally attainable. As shown on Figure 7(a), the broadband noise design objective is reduced by approximately $47 \%$. Since no aerodynamic constraints are imposed in this case, the shape optimization does result in a 5\% penalty on the lift-to-drag ratio. The aerodynamic and aeroacoustic design objectives are clearly competing for trailing edge noise minimization. Aeroacoustic design optimizations should be performed with appropriate design constraints so as to prevent degradation of aerodynamic performance - this is explored in the next subsection. The baseline NACA0012 profile and the optimized profile are compared on Figure 7(b). The optimizer has reduced the curvature of the upper surface significantly and rotated the trailing edge upwards. The effect of the optimization on the broadband noise source is visualize on Figure 8, showing the Frobenius-norm of the time-averaged Lighthills stress tensor in the trailing-edge region. Clearly, the strong broadband noise source present in the wake region of the baseline NACA0012 profile has been significantly reduced. In particular, the peak broadband noise source has been reduced by approximately $60 \%$. Efforts are underway to verify this first-stage optimization result by an aeroacoustic simulation in which the turbulent flow is computed by a scale-resolving method such as large-eddy simulation.

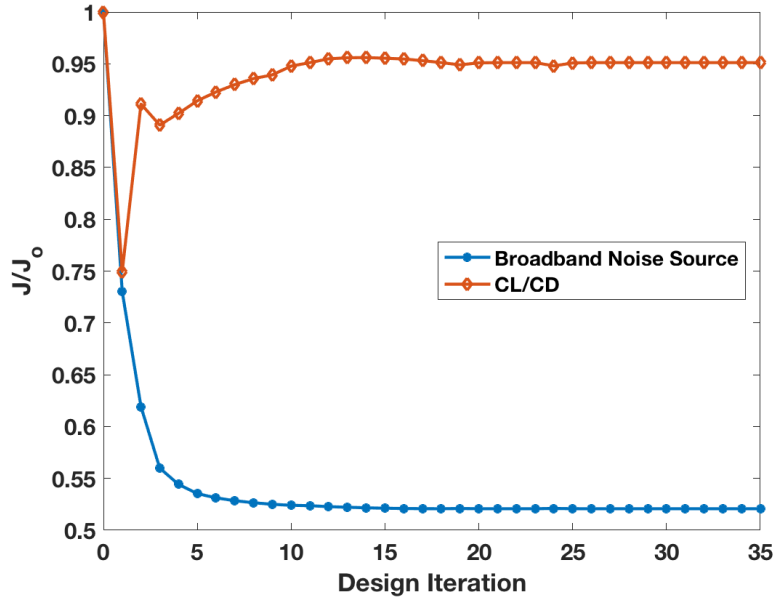

(a) Optimization History (Unconstrained)

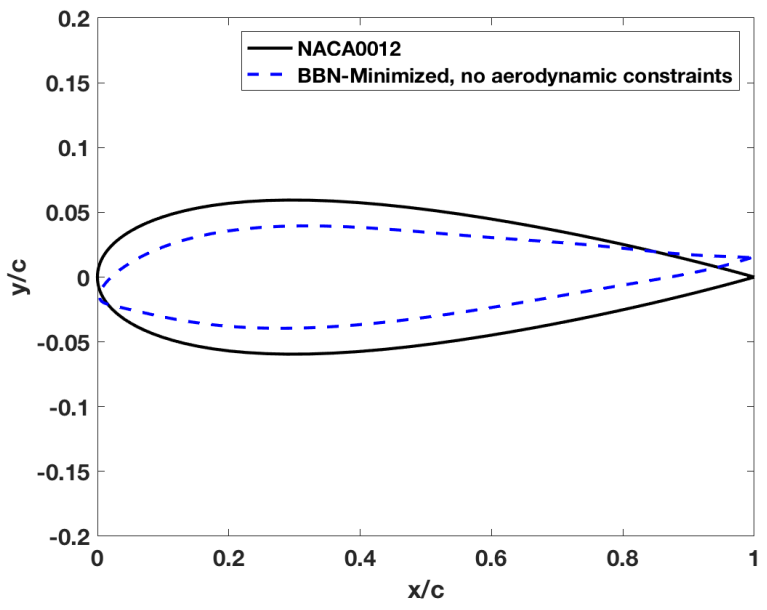

(b) Design Comparison

Figure 7: (a) Convergence of the broadband noise design objective and (b) comparison between the baseline NACA0012 design and optimized design obtained without aerodynamic constraints

\section{III.D. Broadband Noise Source Minimization, Lift-to-Drag Ratio Constrained}

Results in Section III.C clearly indicate that the aerodynamic and aeroacoustic design objectives are competing, in that a shape change that results in a reduction in the broadband noise source also gives rise to the deterioration of aerodynamic performance. In this subsection, a constrained shape optimization is performed whereby the lift-to-drag ratio is constrained to its baseline level. This leads to an additional adjoint solution at each design iteration. As shown on Figure 9(a), the broadband noise design objective is reduced by a much more moderate amount of $28 \%$, in constrast to the $47 \%$ achieved in the unconstrained optimization in the previous subsection. The lift-to-drag ratio, however is constrained at its baseline value. It should be noted that to prevent the 5\% penalty on the lift-to-drag ratio from the unconstrained case, the attainable reduction of the broadband noise source is reduced from $47 \%$ to $28 \%$ in the constrained case. Figure 9(b) compares the baseline NACA0012 design and optimized designs with and without a constraint on the lift-to-drag ratio. As one would expect, the shape changes corresponding to the constrained optimization are far more subtle. The effect of the optimization on the broadband noise source is visualize on Figure 10, showing the Frobenius-norm of the time-averaged Lighthills stress tensor in the trailing-edge region. 


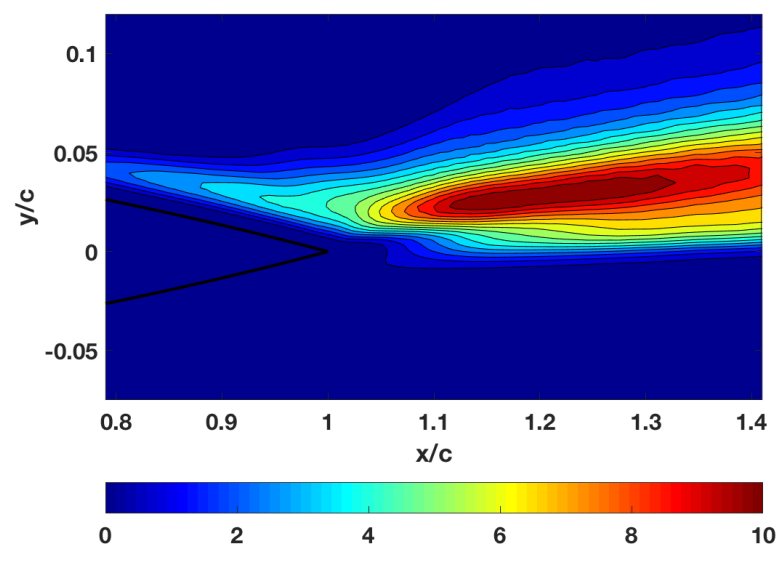

(a) Baseline

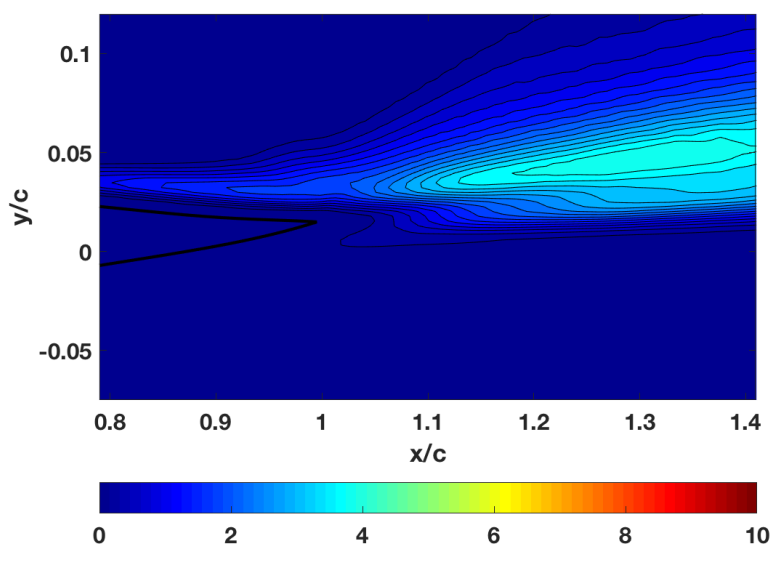

(b) Optimized, without aerodynamic constraints

Figure 8: Frobenius norm of the time-averaged Lighthills stress tensor in the trailing-edge region

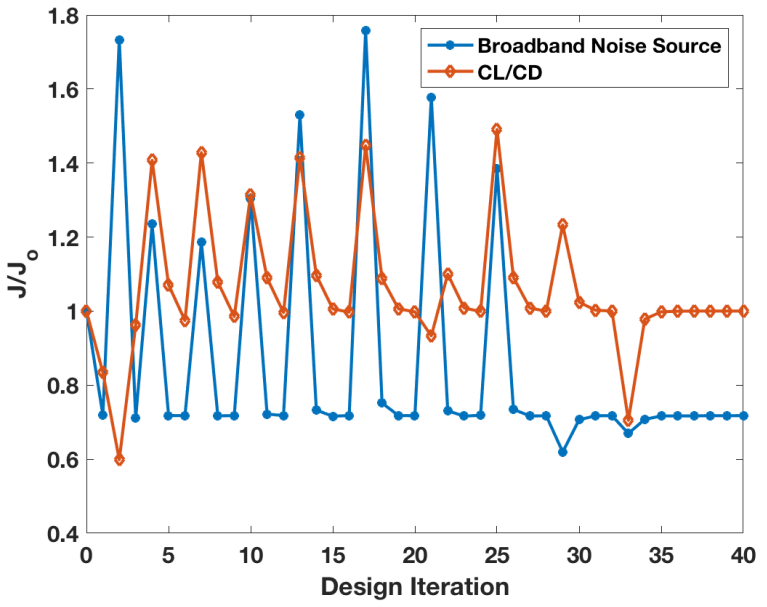

(a) Optimization History (Constrained)

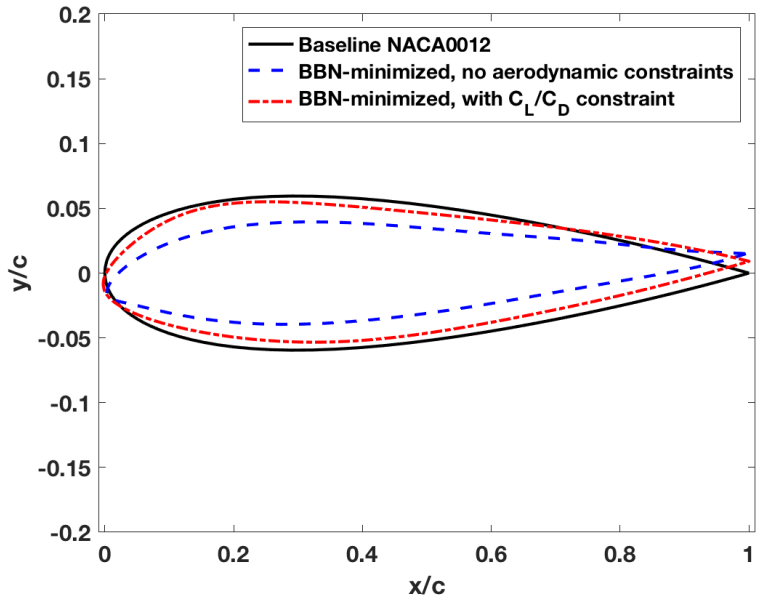

(b) Design Comparison

Figure 9: (a) Convergence of the broadband noise design objective and (b) comparison between the baseline NACA0012 design and optimized designs with and without a constraint on the lift-to-drag ratio 


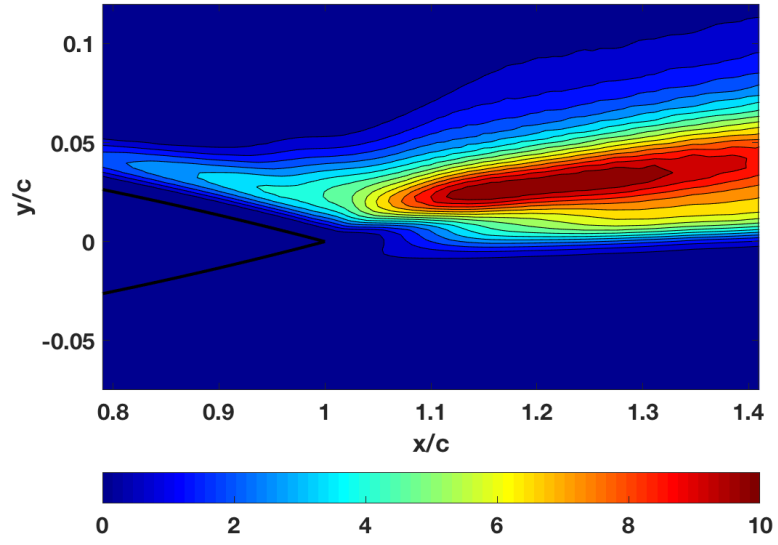

(a) Baseline

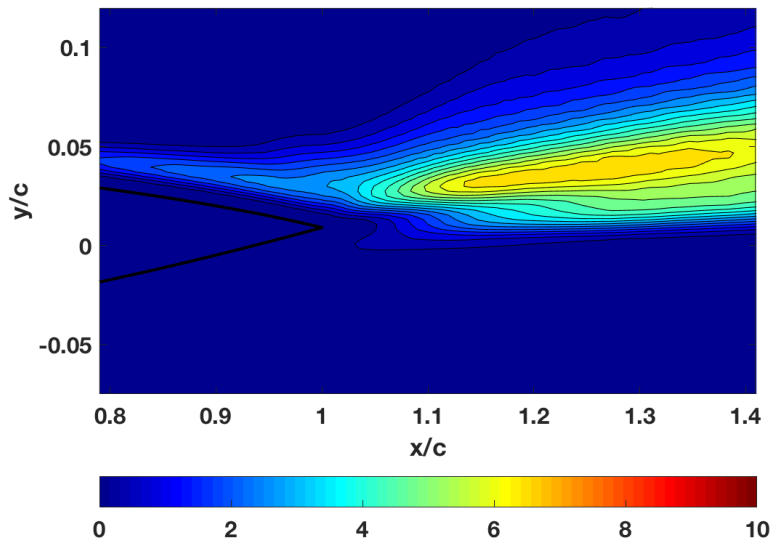

(b) Optimized, lift-to-drag ratio constrained

Figure 10: Frobenius norm of the time-averaged Lighthills stress tensor in the trailing-edge region

\section{Conclusion and Outlook}

In this study, we develop a coupled-adjoint RANS-SNG framework in the SU2 solver, constructed in a manner similar to the coupled URANS-FWH noise minimization framework demonstrated in our previous studies. ${ }^{7,26}$ The adjoint-based RANS-SNG framework proposed here not only avoids the regularization problem that plagues the adjoint solutions for scale-resolving simulations, but more importantly it also significantly lowers the computational cost and leads to a faster turn-around time for the initial design evaluation phase. Preliminary results show that the RANSSNG method can efficiently provide broadband noise level assessment for various configurations without resorting to computationally prohibitive scale-resolving simulations. In addition, current results also show that the coupled adjoint-RANS-SNG solver in SU2, developed on the basis of algorithmic differentiation is highly accurate. Shape optimizations are performed on the basis of such coupled-sensitivities to minimize the broadband noise source in the trailing edge of a NACA0012 airfoil profile both with and without an aerodynamic design constraint. Efforts are underway to compare the baseline and optimized geometries by aeroacoustic simulations in which the turbulent flow is computed by a large-eddy simulation.

In the near future, the existing framework will be applied to optimize the wingtip of a 3-D NACA0012 airfoil section ${ }^{27}$ of a finite span at $12^{\circ}$ angle of attack, $R e=1.8 \times 10^{6}$ and $M=0.175$, as a first step towards flap sideedge noise minimization via shape optimization. It is known from previous work that the turbulent flow around such blunt wingtip is similar to that of the flap side-edge. The wingtip shape will be deformed by the existing free-form deformation capability in SU2. Aeroacoustic simulations based on the hybrid RANS-LES method will be performed on the baseline and optimized configurations to verify whether the optimal design obtained by the adjoint-based RANS-SNG approach is truly superior in terms of its broadband noise level.

\section{References}

${ }^{1}$ Lockard, D. and Lilley, G., "The airframe noise reduction challenge," NASA/TM-2004-213013, 2004.

${ }^{2}$ Colonius, T. and Lele, S. K., "Computational Aeroacoustics: Progress on Nonlinear Problems of Sound Generation," Progress in Aerospace Sciences, Vol. 40, 2004, pp. 345-416.

${ }^{3}$ Lele, S. K. and Nichols, J. W., “A second golden age of aeroacoustics?” Philosophical Transactions of the Royal Society of London, Vol. 372 , 2014.

${ }^{4} J a m e s o n$, A., "Aerodynamic Design via Control Theory," Journal of Scientific Computing, Vol. 3, No. 3, 1988, pp. $233-260$.

${ }^{5}$ Pironneau, O., "On Optimum Design in Fluid Mechanics," Journal of Fluid Mechanics, Vol. 64, No. 1, 1974, pp. 97-110.

${ }^{6}$ Blonigan, P., Chen, R., Wang, Q., and Larsson, J., “Towards adjoint sensitivity analysis of statistics in turbulent flow simulation." In Proceedings of the Summer Program (2012), 2012.

${ }^{7}$ Zhou, B. Y., Albring, T., Gauger, N. R., Ilario, C. R., Economon, T. D., and Alonso, J. J., "Reduction of Airframe Noise Components Using a Discrete Adjoint Approach," AIAA-2017-3658, 2017.

${ }^{8}$ Béchara, W., Bailly, C., Lafon, P., and Candel, S., "Stochastic approach to noise modeling for free turbulent flows," AIAA Journal, Vol. 32(3), 1994, pp. 455-464.

${ }^{9}$ Bailly, C. and Juvé, D., “A stochastic approach to compute subsonic noise using linearized Euler’s equations,” AIAA-1999-1872, 1999. 
${ }^{10}$ Ewert, R. and Schröder, W., "On the Simulation of Trailing Edge Noise with a Hybrid LES/APE Method," Journal of Sound and Vibration, Vol. 270, 2004, pp. 509-524.

${ }^{11}$ Billson, M., Eriksson, L.-E., and Davidson, L., "Jet noise modeling using synthetic anisotropic turbulence," AIAA Paper, 2004, pp. 20043028.

${ }^{12}$ Casalino, D. and Barbarino, M., "A stochastic method for airfoil self-noise computation in frequency-domain,” AIAA-2010-3884, 2010.

${ }^{13}$ di Francescantonio, P. ., Ferrante, P., Deconinck, T., and Hirsch, C., "Assessment of SNGR Method for Robust and Efficient Simulation of Flow Generated Noise," AIAA-2013-2264, 2013.

${ }^{14}$ Yao, H., Davidson, L., Peng, S., Eriksson, L., Barbarino, M., Capizzano, F., and Mingione, G., "Assessment of Flap Side-Edge Fence Noise using SNGR Method," AIAA Paper 2015-2224, 2015.

${ }^{15}$ Yao, H., Davidson, L., Peng, S., Barbarino, M., Capizzano, F., and Mingione, G., "Assessment of Conceptual Noise Reduction Devices for A Main Landing Gear using SNGR Method," AIAA Paper 2015-2692, 2015.

${ }^{16}$ Hogan, R., "Fast reverse-mode automatic differentiation using expression templates in C++," Transactions on Mathematical Software, Vol. 40, 2014, pp. 1-16.

${ }^{17}$ Hovland, P., Mohammadi, B., and Bischof, C., "Automatic Differentiation and Navier-Stokes Computations," Computation Methods for Optimal Design and Control, Vol. 24, 1998, pp. 265-284.

${ }^{18}$ Gauger, N. R., Walther, A., Moldenhauer, C., and Widhalm, M., "Automatic Differentiation of an Entire Design Chain for Aerodynamic Shape Optimization," Notes on Numerical Fluid Mechanics and Multidisciplinary Design, Vol. 96, 2007, pp. 454-461.

${ }^{19}$ Nemili, A., Özkaya, E., Gauger, N. R., Kramer, F., Höll, T., and Thiele, F., "Optimal Design of Active Flow Control for a Complex High-Lift Configuration," AIAA-2014-2515, 2014.

${ }^{20}$ Palacios, F., Economon, T. D., Aranake, A. C., Copeland, S. R., Lonkar, A. K., Lukaczyk, T. W., Manosalvas, D. E., Naik, K. R., Padron, A. S., Tracey, B., Variyar, A., and Alonso, J. J., "Stanford University Unstructured (SU2): Open-source analysis and design technology for turbulent flows," AIAA Journal, Vol. 54, 2016, pp. 828-846.

${ }^{21}$ Zhao, X. and He, G. W., "Space-time correlations of fluctuating velocities in turbulent shear flows," Phys Rrev. E, Vol. 79, No. 4, 2009, pp. 046316. 1872.

${ }^{22}$ Bailly, C. and Juvé, D., “A stochastic approach to compute subsonic noise using linearized Euler's equations,” AIAA Paper, 1999, pp. 1999-

${ }^{23}$ Sagebaum, M., Albring, T., and Gauger, N. R., "High-Performance Derivative Computations using CoDiPack," arXiv preprint arXiv:1709.07229, 2017.

${ }^{24}$ Hicks, R. M. and Henne, P. A., "Wing Design by Numerical Optimization," Journal of Aircraft, Vol. 15, 1978, pp. 407-412. 160.

${ }^{25}$ Sederberg, T. W. and Parry, S. R., "Free-form deformation of solid geometric models," SIGGRAPH Comput. Graph., Vol. 20, 1986, pp. 151-

${ }^{26}$ Zhou, B. Y., Albring, T., Gauger, N. R., Ilario, C. R., Economon, T. D., and Alonso, J. J., "A Discrete Adjoint Approach for Jet-Flap Interaction Noise Reduction," AIAA-2017-0130, 2017.

${ }^{27}$ Imamura, T., Enomoto, S., and Yamamoto, K., "Noise Simulation around NACA0012 Wingtip using Large Eddy Simulation,” Transactions of the Japan Society for Aeronautical and Space Sciences, Vol. 55, 2012, pp. 214-221. 